Article

\title{
Camptocormia as a Novel Phenotype in a Heterozygous POLG2 Mutation
}

\author{
Diana Lehmann Urban ${ }^{1, *}$, Leila Motlagh Scholle ${ }^{2}$, Kerstin Alt ${ }^{3}$, Albert C. Ludolph ${ }^{1}$ and \\ Angela Rosenbohm 1 \\ 1 Department of Neurology, Ulm University, 89081 Ulm, Germany \\ 2 Department of Neurology, University of Halle/S., 06120 Halle, Germany \\ 3 Genetikum, 89231 Neu-Ulm, Germany \\ * Correspondence: diana.lehmann@rku.de; Tel.: +49-(0)731-1771201; Fax: +49-(0)731-1771202
}

Received: 23 December 2019; Accepted: 23 January 2020; Published: 26 January 2020

\begin{abstract}
Mitochondrial dysfunction is known to play a key role in the pathophysiological pathway of neurodegenerative disorders. Nuclear-encoded proteins are involved in mtDNA replication, including DNA polymerase gamma, which is the only known replicative mtDNA polymerase, encoded by nuclear genes Polymerase gamma 1 (POLG) and Polymerase gamma 2 (POLG2). POLG mutations are well-known as a frequent cause of mitochondrial myopathies of nuclear origin. However, only rare descriptions of POLG2 mutations leading to mitochondriopathies exist. Here we describe a 68-year-old woman presenting with a 20-year history of camptocormia, mild proximal weakness, and moderate CK increase. Muscle histology showed COX-negative fibres. Genetic analysis by next generation sequencing revealed an already reported heterozygous c.1192-8_1207dup24 mutation in the POLG2 gene. This is the first report on a POLG2 mutation leading to camptocormia as the main clinical phenotype, extending the phenotypic spectrum of POLG2 associated diseases. This underlines the broad phenotypic spectrum found in mitochondrial diseases, especially in mitochondrial disorders of nuclear origin.
\end{abstract}

Keywords: mitochondrial myopathy; polymerase gamma 2 (POLG2); camptocormia; mutations of nuclear origin

\section{Introduction}

Mitochondrial diseases are associated with a wide spectrum of different clinical phenotypes, ranging from mild to severe [1]. They can either be caused by mutations in the mitochondrial DNA (mtDNA) itself or due to mutations of nuclear origin: (I) nuclear genes encoding for enzymes involved in mitochondrial nucleotide synthesis (TK2, SUCLA2, SUCLG1, RRM2B, DGUOK, and TYMP) or (II) those required for mtDNA replication (POLG and TWNK) [2]. mtDNA replication is accomplished by DNA polymerase gamma, a heterotrimer consisting of one catalytic subunit of DNA polymerase encoded by POLG (alternative gene symbol: POLG1) and a dimer of accessory subunits encoded by POLG2, a processivity factor for the DNA polymerase [3-5]. POLG mutations are well-known to cause either autosomal dominant (ad) or recessive (ar) mutations associated with a broad phenotypic spectrum and may account for up to $25 \%$ of all adult presentations of mitochondrial diseases [6]. Since the first publication of POLG mutations leading to mitochondrial myopathies [7], over hundred different $P O L G$ mutations have been identified with a wide range of phenotypical presentations. However, only rare descriptions of POLG2 mutations leading to mitochondriopathies exist [5,8-11]. Camptocormia (paravertebral muscle weakness with disabling flexion of the spine in upright position) has been described in case reports and case series in association with Parkinsonian disorders, dystonia, and infrequently in functional neurologic disorders [12]. Only in a few cases, camptocormia has been 
reported in association with myopathic and/or mitochondrial defects [13-19]. Here, we present a novel phenotype in an already reported heterozygous POLG2 mutation [2] with the clinical leading symptom of camptocormia, extending the phenotypic spectrum of POLG2 related mitochondrial myopathies.

\section{Materials and Methods}

\subsection{Clinical Description}

A 68-year-old caucasian female presented with a lumbar kinking camptocormia (Figure 1A), developing over the last 20 years. Walking was only possible using the help of walking sticks, self-erecting was only possible for a few seconds. Laboratory testing showed a mild CK increase (308 U/L, controls < $180 \mathrm{U} / \mathrm{L}$ ). Her medical history included chronic pain syndrome, left convex lumbar scoliosis, arterial hypertension, hypercholesterolemia, polyneuropathy and arthrosis of the small finger joints. Clinical examination revealed proximal weakness of the upper limbs (MRC 4/5) and pronounced weakness of the paravertebral lumbar spine muscles with a lumbal camptocormia of $30-40^{\circ}$ deviation from the vertical orientation. No ptosis or ophthalmoplegia were found. Deep tendon reflexes were normal and symmetrical. No Babinski sign. Spinal MRI showed a significant fatty degeneration of the paravertebral lumbar spine muscles, no evidence of muscle edema or pathologic CM-uptake indicating florid disease activity (Figure 1B). Electromyography showed chronic neurogenic changes. The patient has three healthy children, with no history of muscle disease, however a neurological examination has been declined. The parents of the index- patient died at higher age without evidence for neuromuscular disorder.

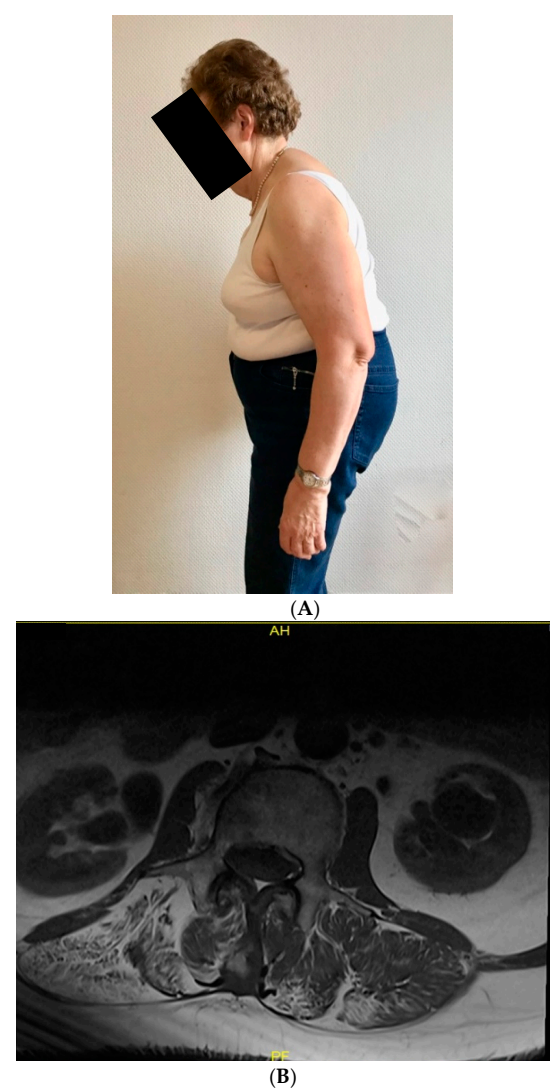

Figure 1. (A) Camptocormia in the patient as the main clinical symptom: upright standing is only possible with bended knees, indicating the severity of camptocormia and (B) transversal T1- weighted spinal MRI showing a significant fatty degeneration of the paravertebral lumbar spine muscles. 


\subsection{Muscle Histopathology}

Cyrostat sections were cut from transversely orientated muscle blocks from the lumbal erector spinae muscle and subjected to standard histological and histochemical analysis including COX, succinate dehydrogenase (SDH), and COX-SDH oxidative enzyme reactions.

\subsection{Activities of Respiratory Chain Complexes}

Activities of respiratory chain complexes were determined spectrophotometrically according to standard protocols [20].

\subsection{Next Generation Sequencing}

To analyze the coding areas as well as $25 \mathrm{bp}$ of flanking intronic sequence of 252 genes associated with different types of myopathies including genes for nuclear encoded mitochondriopathies, genomic DNA was extracted from a patient's EDTA-blood sample with the FlexiGene DNA Kit following the manufacturer's protocol (http://www.qiagen.com/). Subsequently, target enrichment was performed with the HaloPlex Target Enrichment System (Protocol Version D, Agilent Technologies, Santa Clara, CA, USA) with an input of 50 ng DNA. After library pooling, next generation sequencing was performed on the Illumina NextSeq 500 System (Illumina, San Diego, CA, USA) with 150 base pair (bp) paired-end reads. The megSAP pipeline was used for bioinformatical analysis [21]. Adapter sequences were removed with SeqPurge [22], trimmed reads were aligned with BWA-MEM [23] to the human reference genome hg19/GRCh37 and improved by indel realignment with $A B R A$ [24], yielding an average coverage of 700 reads. Variants were called with freebayes [25]. All regions that lacked sufficient read depth $(<5$ reads) were excluded from analysis. All variants with a percentage share of less than $10 \%$ were initially not considered for further analysis. Variant annotation was performed by SnpEff [26]. To obtain allelic frequencies, all variants were annotated with several public databases such as ExAC [27] and 1000 genomes [28] as well as our in-house database and databases for clinical significance like ClinVar [29]. For the prioritization of potentially clinically relevant variants, GSvar (part of ngs-bits [30]) was used to remove all variants with allelic frequencies higher than $1 \%$ in any annotated public database or common occurrence in our in-house database $(>20)$ unless they were labeled as pathogenic. Nomenclature of the Human Genome Variation Society (HGVS) was applied to describe the identified variants [31].

\section{Results}

\subsection{Clinical Findings and Muscle Biopsy}

The combination of clinical examination, showing proximal weakness of the upper limbs (MRC 4/5), together with camptocormia (Figure 1A,B) and mild CK increase (308 U/L, controls $<180 \mathrm{U} / \mathrm{L}$ ) lead to the differential diagnosis of mitochondrial and other metabolic disorders.

A lumbal erector spinae muscle biopsy showed myopathic changes, including central nuclei, endomysial fibrosis, elevated fiber caliber variation and COX-negative muscle fibers, so as occasional cores. Interestingly, no evidence for ragged red fibres (RRF) was found (Figure 2). Due to the findings of COX-negative fibers and cores, genetic testing has been initiated. 

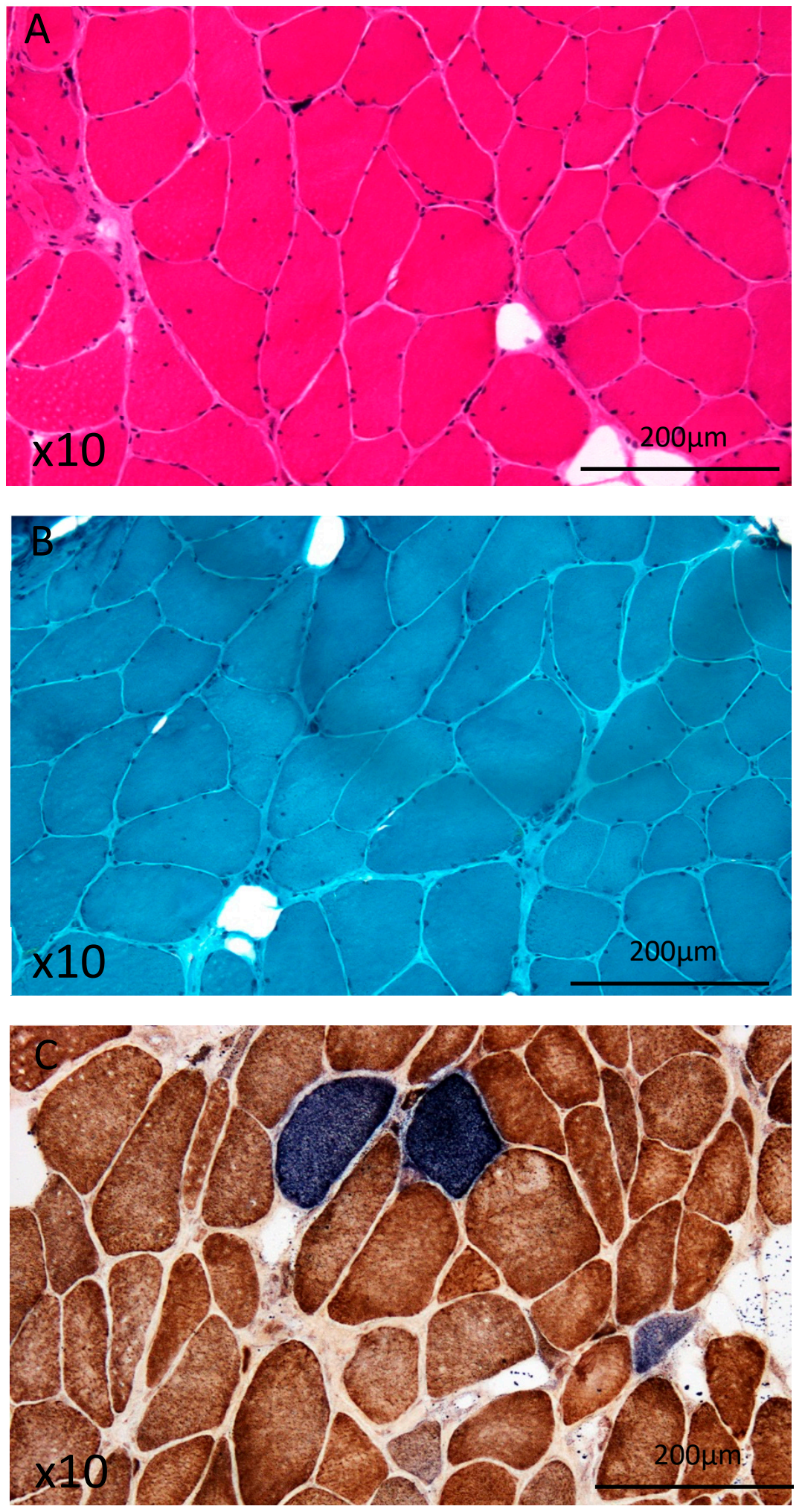

Figure 2. Histochemical findings: (A) Hematoxylin and eosin staining, (B) Gomori trichrome staining and (C) cytochrome c oxidase (COX)-SDH histochemistry revealing numerous COX-deficient (blue reaction product) fibres. 


\subsection{Activities of Respiratory Chain Complexes}

Measurements of respiratory chain activities showed decreased activities of respiratory chain complexes I, II + III, and IV (Table 1). Furthermore, citrate synthase was noticeably decreased in patient's muscle, too.

Table 1. Enzyme activity of respiratory chain complexes, showing decreased activities of respiratory chain complexes I, II + III and IV, as well as decreased citrate synthase activity in the patient muscle.

\begin{tabular}{ccc}
\hline \multirow{2}{*}{ Respiratory Chain Complexes } & \multicolumn{2}{c}{ Enzyme Activity (U/g Tissue) } \\
\cline { 2 - 3 } & Patient & Controls $(\boldsymbol{n}=\mathbf{2 0})$ Mean \pm SD (Range) \\
\hline Complex I & 0.125 & $0.9 \pm 0.6(0.35-2.5)$ \\
Complexes II + III & 0.48 & $1.8 \pm 0.8(0.8-2.6)$ \\
Complex IV (COX) & 1.8 & $10.3 \pm 1.5(8.2-12.4)$ \\
Citrate synthase & 0.34 & $8.4 \pm 2.7(4.0-11.2)$ \\
\hline
\end{tabular}

\subsection{Genetic Analysis}

Due to central cores in oxidative staining, gene panel analysis using next generation sequencing (NGS) was initiated without prior mtDNA sequencing. NGS was performed according to standard protocols and showed no pathological mutations in 251 myopathy-related genes including RYR1, POLG and other nuclear encoded mitochondrial genes (supplementary materials). Hence, the possibility that the camptocormia resulted from mutations in any of these 251 genes was ruled out. Analysis of POLG2 gene revealed an already reported heterozygous c.1192-8_1207dup24 mutation [8]. Unfortunately, the analysis of further patient material or from family members has been declined.

\section{Discussion}

Mitochondrial diseases are known to show a broad phenotypic spectrum, like varying age-of-onset range. The first reported POLG2 case was documented in a patient with adult-onset autosomal dominant (ad) progressive external ophthalmoplegia (PEO) as well as COX-deficient muscle fibres and multiple deletions in the mtDNA [9]. Later on, three pediatric- onset autosomal dominant disease cases with varying phenotypes (one young adult with adPEO in late teens and two unrelated infants developing hypotonia, seizures and liver disease) were reported [11]. An infant with fulminant hepatic failure and mitochondrial DNA depletion caused by a homozygous POLG2 missense variant has been reported [5]. A Belgian pedigree presented with adult-onset cerebellar ataxia, axonal peripheral ataxic neuropathy and tremor in variable combination with parkinsonism, seizures, cognitive decline and opthalmoplegia [10]. The first patient reported with the heterozygous c.1192-8_1207dup24 mutation clinically presented with late-onset ptosis and myopathy [8]. These findings clearly underline the wide phenotypic spectrum, especially in mitochondrial diseases due to nuclear origin, e.g., POLG mutations.

Here, we report a novel phenotype caused by an already known POLG2 mutation. Camptocormia has already been, however only rarely reported in association with myopathic and mitochondrial defects [13-19]. Until now, camptocormia has not been described in the phenotypic spectrum of mitochondrial myopathies caused by POLG2 mutations.

Gomori trichrome staining has been postulated as the most sensible and reliable method for detection of mitochondrial abnormalities [32]. Recently, 19 patients harboring POLG mutations were studied [33]. Histochemical abnormalities were found in 17/19 cases: cox-negative fibers in 13 cases $(68.4 \%)$ and ragged red fibers in 12 cases (63.2\%) [33]. Interestingly, in the presented case, muscle histology showed no evidence for ragged-red fibres (RRF). This is consistent with a recent study, in which 11 patients with multiple deletions due to POLG mutations were analyzed according to frequency of histopathological changes [34] and only $2 \%$ of fibres from patients with POLG mutations were classified as RRF. However, in this study it has not been clarified which muscle has been taken for biopsy. 
The only slight evidence for mitochondrial dysfunction shown in histopathology in the presented case is consistent with the mild clinical phenotype, which coincides with the recently reported patient with the same mutation [8]. The reported mutation was found to cause missplicing and loss of exon 7 in myoblast cDNA due to the $24 \mathrm{bp}$ insertion into exon 7 [8]. However, in none of the reported POLG2 mutations, camptocormia has been described. Recently, limb muscle biopsy has been recommended as a diagnostic procedure in camptocormia [35]. However, in the presented case, a biopsy of the lumbal erector spinae muscle was performed.

The evidence of cores in muscle histopathology is usually indicational for central core disease (CCD), a congenital myopathy caused by mutations in the gene encoding ryanodine receptor type- 1 (RYR1) with muscle weakness defined pathologically by the presence of extensive areas in muscle fibres that are devoid of oxidative enzyme activity ("central cores") [36-38]. Up to date, evidence of cores in patients with mitochondrial diseases due to either mutations of the mtDNA or due to nuclear origin have not been reported. Interestingly, muscle histopathology of the patient presented in this study showed evidence of occasional cores.

Our finding substantially adds to the spectrum of differential diagnostic considerations in patients with camptocormia and histopathological evidence of cores. This strongly underlines the importance of skeletal muscle biopsy as a major diagnostic tool in patients with suspected mitochondrial disease.

Supplementary Materials: Supplementary materials can be found at http://www.mdpi.com/2075-4418/10/2/68/s1.

Author Contributions: Preparation of manuscript and interpretation of the data: D.L.U. and A.R.; experimental work: L.M.S. and K.A.; clinical and histopathological workup: D.L.U. and A.R.; supervision and critical review: A.C.L. and A.R. All authors have read and agreed to the published version of the manuscript.

Funding: D.L.U. is funded by the Hertha-Nathorff-Programm (HNP) of the Ulm Medical University, Germany. D.L.U. and L.M.S. are members of the German mitoNET funded by the German Ministry of Education and Research.

Acknowledgments: We acknowledge the excellent technical assistance of Martina Leis (Muscle laboratory of the Department of Neurology, University Hospital Ulm, Germany) and the excellent work of Christoph Schmidt (Department of Bioinformatics, Genetikum, Neu-Ulm, Germany). We acknowledge the financial support within the funding programme Open Access Publishing by the German Research Foundation (DFG).

Conflicts of Interest: The authors declare no conflict of interest.

\section{References}

1. Lehmann, D.; Schubert, K.; Joshi, P.R.; Baty, K.; Blakely, E.L.; Zierz, S.; Taylor, R.W.; Deschauer, M. A novel $\mathrm{m} .7539 \mathrm{C}>\mathrm{T}$ point mutation in the mt-tRNA(Asp) gene associated with multisystemic mitochondrial disease. Neuromuscul. Disord. 2015, 25, 81-84. [CrossRef] [PubMed]

2. El-Hattab, A.W.; Scaglia, F. Mitochondrial DNA depletion syndromes: Review and updates of genetic basis, manifestations, and therapeutic options. Neurotherapeutics 2013, 10, 186-198. [CrossRef] [PubMed]

3. Da Pozzo, P.; Cardaioli, E.; Rubegni, A.; Gallus, G.N.; Malandrini, A.; Rufa, A.; Battisti, C.; Carluccio, M.A.; Rocchi, R.; Giannini, F.; et al. Novel POLG mutations and variable clinical phenotypes in 13 Italian patients. Neurol. Sci. 2017, 38, 563-570. [CrossRef] [PubMed]

4. Copeland, W.C. Defects of mitochondrial DNA replication. J. Child. Neurol. 2014, 29, 1216-1224. [CrossRef]

5. Varma, H.; Faust, P.L.; Iglesias, A.D.; Lagana, S.M.; Wou, K.; Hirano, M.; Di Mauro, S.; Mansukani, M.M.; Hoff, K.E.; Nagy, P.L.; et al. Whole exome sequencing identifies a homozygous POLG2 missense variant in an infant with fulminant hepatic failure and mitochondrial DNA depletion. Eur. J. Med. Genet. 2016, 59, 540-545. [CrossRef]

6. Stewart, J.D.; Tennant, S.; Powell, H.; Pyle, A.; Blakely, E.L.; He, L.; Hudson, G.; Roberts, M.; du Plessiset, D.; Gow, D.; et al. Novel POLG1 mutations associated with neuromuscular and liver phenotypes in adults and children. J. Med. Genet. 2009, 46, 209-214. [CrossRef]

7. Van Goethem, G.; Dermaut, B.; Löfgren, A.; Martin, J.-J.; van Broeckhoven, C. Mutation of POLG is associated with progressive external ophthalmoplegia characterized by mtDNA deletions. Nat. Genet. 2001, 28, 211-212. [CrossRef] 
8. Walter, M.C.; Czermin, B.; Muller-Ziermann, S.; Bulst, S.; Stewart, J.D.; Hudson, G.; Schneiderat, P.; Abicht, A.; Holinski-Feder, E.; Lochmüller, H.; et al. Late-onset ptosis and myopathy in a patient with a heterozygous insertion in POLG2. J. Neurol. 2010, 257, 1517-1523. [CrossRef]

9. Longley, M.J.; Clark, S.; Man, C.Y.W.; Hudson, G.; Durham, S.E.; Taylor, R.W.; Nightingale, S.; Turnbull, D.M.; Copeland, W.C.; Chinnery, P.F. Mutant POLG2 disrupts DNA polymerase gamma subunits and causes progressive external ophthalmoplegia. Am. J. Hum. Gene 2006, 78, 1026-1034. [CrossRef]

10. Van Maldergem, L.; Besse, A.; De Paepe, B.; Blakely, E.L.; Appadurai, V.; Humble, M.M.; Piard, J.; Craig, K.; He, L.; Hella, P.; et al. POLG2 deficiency causes adult-onset syndromic sensory neuropathy, ataxia and parkinsonism. Ann. Clin. Transl. Neurol. 2017, 4, 4-14. [CrossRef]

11. Young, M.J.; Longley, M.J.; Li, F.-Y.; Kasiviswanathan, R.; Wong, L.-J.; Copeland, W.C. Biochemical analysis of human POLG2 variants associated with mitochondrial disease. Hum. Mol. Genet. 2011, 20, 3052-3066. [CrossRef] [PubMed]

12. Ali, F.; Matsumoto, J.Y.; Hassan, A. Camptocormia: Etiology, diagnosis, and treatment response. Neurol. Clin. Pract. 2018, 8, 240-248. [CrossRef] [PubMed]

13. Sakiyama, Y.; Okamoto, Y.; Higuchi, I.; Inamori, Y.; Sangatsuda, Y.; Michizono, K.; Watanabe, O.; Hatakeyama, H.; Goto, Y.; Arimura, K.; et al. A new phenotype of mitochondrial disease characterized by familial late-onset predominant axial myopathy and encephalopathy. Acta Neuropathol. 2011, 121, 775-783. [CrossRef]

14. Delcey, V.; Hachulla, E.; Michon-Pasturel, U.; Queyrel, V.; Hatron, P.Y.; Boutry, N.; Lemaitre, V.; Vanhille, P.; Serratrice, J.; Disdier, P.; et al. Camptocormia: A sign of axial myopathy. Report of 7 cases. Rev. Med. Interne. 2002, 23, 144-154. [CrossRef]

15. Gomez-Puerta, J.A.; Peris, P.; Grau, J.M.; Martinez, M.A.; Guañabens, N. Camptocormia as a clinical manifestation of mitochondrial myopathy. Clin. Rheumatol. 2007, 26, 1017-1019. [CrossRef] [PubMed]

16. Poullin, P.; Daumen-Legre, V.; Serratrice, G. Camptocormia in the elderly patient: Myopathy or muscular dystonia? Rev. Rhum. Ed. Fr. 1993, 60, 159-161.

17. Schabitz, W.R.; Glatz, K.; Schuhan, C.; Sommer, C.; Berger, C.; Schwaninger, M.; Hartmann, M.; Hilmar Goebel, H.; Meinck, H.M. Severe forward flexion of the trunk in Parkinson's disease: Focal myopathy of the paraspinal muscles mimicking camptocormia. Mov. Disord. 2003, 18, 408-414. [CrossRef]

18. Serratrice, G.; Pouget, J.; Pellissier, J.F. Bent spine syndrome. J. Neurol. Neurosurg. Psychiatry 1996, 60, 51-54. [CrossRef]

19. Reimann, J.; Lehmann, D.; Hardy, S.A.; Falkous, G.; Knowles, C.V.Y.; Jones, R.L.; Kunz, W.S.; Taylor, R.W.; Kornblum, C. Camptocormia and shuffling gait due to a novel MT-TV mutation: Diagnostic pitfalls. Neurol. Genet. 2017, 3, e147. [CrossRef]

20. Gellerich, F.N.; Deschauer, M.; Chen, Y.; Müller, T.; Neudecker, S.; Zierz, S. Mitochondrial respiratory rates and activities of respiratory chain complexes correlate linearly with heteroplasmy of deleted mtDNA without threshold and independently of deletion size. Biochim. Biophys. Acta 2002, 1556, 41-52. [CrossRef]

21. megSAP_A Medical Genetics Sequence Analysis Pipeline. Available online: https://github.com/imgag/ megSAP (accessed on 25 January 2020).

22. Sturm, M.; Schroeder, C.; Bauer, P. SeqPurge: Highly-sensitive adapter trimming for paired-end NGS data. BMC Bioinform. 2016, 17, 208. [CrossRef] [PubMed]

23. Li, H. Aligning sequence reads, clone sequences and assemblycontigs with BWA-MEM. arXiv 2013, arXiv:1303.3997 [q-bio.GN].

24. Mose, L.E.; Wilkerson, M.D.; Hayes, D.N.; Perou, C.M.; Parker, J.S. ABRA: Improved coding indel detection via assembly-based realignment. Bioinformatics 2014, 30, 2813-2815. [CrossRef] [PubMed]

25. Garrison, E.; Marth, G. Haplotype-based variant detection from short-read sequencing. arXiv 2012, arXiv:1207.3907 [q-bio.GN].

26. Cingolani, P.; Platts, A.; Wang, L.L.; Coon, M.; Nguyen, T.; Wang, L.; Land, S.J.; Lu, X.; Ruden, D.M. A program for annotating and predicting the effects of single nucleotide polymorphisms, SnpEff: SNPs in the genome of Drosophila melanogaster strain w1118; iso-2; iso-3. Fly (Austin) 2012, 6, 80-92. [CrossRef]

27. Lek, M.; Karczewski, K.J.; Minikel, E.V.; Samocha, K.E.; Banks, E.; Fennell, T.; O’Donnell-Luria, A.H.; Ware, J.S.; Hill, A.J.; Cummings, B.B.; et al. Analysis of protein-coding genetic variation in 60,706 humans. Nature 2016, 536, 285-291. [CrossRef] 
28. Auton, A.; Abecasis, G.R.; Altshuler, D.M.; Durbin, R.M.; Bentley, D.R.; Chakravarti, A.; Clark, A.G.; Donnelly, P.; Eichler, E.E.; Flicek, P.; et al. A global reference for human genetic variation. Nature 2015, 526, 68-74.

29. Landrum, M.J.; Lee, J.M.; Benson, M.; Brown, G.R.; Chao, C.; Chitipiralla, S.; Gu, B.; Hart, J.; Hoffman, D.; Jang, W.; et al. ClinVar: Improving access to variant interpretations and supporting evidence. Nucleic Acids Res. 2018, 46, D1062-D1067. [CrossRef]

30. ngs-bits-Short-read sequencing tools for diagnostics: GSvar. Available online: https://github.com/imgag/ ngs-bits (accessed on 25 January 2020).

31. Den Dunnen, J.T.; Dalgleish, R.; Maglott, D.R.; Hart, R.K.; Greenblatt, M.S.; McGowan-Jordan, J.; Roux, A.F.; Smith, T.; Antonarakis, S.E.; Taschner, P.E. HGVS Recommendations for the Description of SequenceVariants: 2016 Update. Hum. Mutat. 2016, 6, 564-569. [CrossRef]

32. Reichmann, H.; Vogler, L.; Seibel, P. Ragged red or ragged blue fibers. Eur. Neurol. 1996, 36, $98-102$. [CrossRef]

33. Bereau, M.; Anheim, M.; Echaniz-Laguna, A.; Magot, A.; Verny, C.; Goideau-Sevrain, M.; Barth, M.; Amati-Bonneau, P.; Allouche, S.; Ayrignac, X.; et al. The wide POLG-related spectrum: An. integrated view. J. Neurol Sci. 2016, 368, 70-76. [CrossRef] [PubMed]

34. Zierz, C.M.; Joshi, P.R.; Zierz, S. Frequencies of myohistological mitochondrial changes in patients with mitochondrial DNA deletions and the common m.3243A>G point mutation. Neuropathology 2015, 35, 130-136. [CrossRef] [PubMed]

35. Chanson, J.B.; Lannes, B.; Echaniz-Laguna, A. Is deltoid muscle biopsy useful in isolated camptocormia? A prospective study. Eur. J. Neurol. 2016, 23, 1086-1092. [CrossRef]

36. Paolini, C.; Quarta, M.; Wei-LaPierre, L.; Michelucci, A.; Nori, A.; Reggiani, C.; Dirksen, R.T.; Protasi, F. Oxidative stress, mitochondrial damage, and cores in muscle from calsequestrin-1 knockout mice. Skelet. Muscle 2015, 5, 10. [CrossRef] [PubMed]

37. Jungbluth, H. Central core disease. Orphanet. J. Rare Dis. 2007, 2, 25. [CrossRef] [PubMed]

38. Sewry, C.A.; Müller, C.; Davis, M.; Dwyer, J.S.; Dove, J.; Evans, G.; Schröder, R.; Fürst, D.; Helliwell, T.; Laing, N.; et al. The spectrum of pathology in central core disease. Neuromuscul. Disord. 2002, 12, 930-938. [CrossRef]

(C) 2020 by the authors. Licensee MDPI, Basel, Switzerland. This article is an open access article distributed under the terms and conditions of the Creative Commons Attribution (CC BY) license (http://creativecommons.org/licenses/by/4.0/). 